\title{
Buscando los caminos del sector cooperativo y solidario
}

Looking for the paths of the cooperative and solidarity sector

Procurando os caminhos do setor cooperativo e solidário

\section{Néstor Alfonso Rodríguez Espinosa ${ }^{1}$ Miguel Ricardo Dávila Ladrón De Guevara²}

Recibido: 12 de diciembre de 2019

Aprobado: 14 de agosto de 2020

Publicado: 30 de septiembre de 2020

Cómo citar este artículo:

Rodríguez Espinosa, N.A. y Dávila Ladrón De Guevara, M.R. (2020). Buscando los caminos del sector cooperativo y solidario. Cooperativismo \& Desarrollo, 28(118), 1-32. doi: https://doi.org/10.16925/2382-4220.2020.03.08

Artículo de Reflexión. https://doi.org/10.16925/2382-4220.2020.03.08

1 Magister en Ciencias Financieras y de Sistemas, Especialista en Banca. Candidato a doctor en Economía Social de la Universidad de Valencia

Correo electrónico: nestorodriguez2003@yahoo.es

ORCID: https://orcid.org/0000-0002-6525-519X

2 Profesor Emérito, Pontificia Universidad Javeriana. Economista Industrial, Magíster en Desarrollo Rural. Presidente Honorario, Corporación red Unicosol, Socio de DLDG.

Correo electrónico: rdldg23@gmail.com

ORCID: https://orcid.org/0000-0002-3384-9217 


\section{Resumen}

Con este artículo se pretende identificar dónde se ubica la dinámica actual del sector cooperativo y solidario. Para lograr este objetivo se consulta a expertos sobre el escenario en que transita este sector actualmente, revisando el cumplimiento o no de los objetivos estratégicos planteados en el trabajo titulado "Aplicación del modelo prospectivo estratégico al sector cooperativo colombiano. Horizonte: año 2031" publicado en 2014 por el Instituto de Estudios del Ministerio Público. De igual forma, se buscó evidencias en documentos publicados por organismos del Estado y del propio sector. El principal hallazgo de este trabajo se relaciona con la ubicación del sector en un estado similar al de hace cinco años, con algunos avances importantes, pero no suficientes para considerar transformaciones que lleven a escenarios distintos de mayor incidencia en la vida de las regiones en que se desarrolla. Lo anterior nos lleva a concluir que de seguir este estado de las cosas en el sector, su desarrollo será influenciado por la fuerza de las dinámicas externas, bien sea de los movimientos sociales o de la instrumentalización de los gobernantes de turno, como se ha observado históricamente.

Palabras clave: Economía Solidaria; Cooperativismo; Planeación Prospectiva; Organismos de estado, Escenario prospectiva.

P13 Empresas cooperativas

R58 Planificación y política de desarrollo regional

P21 Planificación, coordinación y reforma

\section{Abstract}

This article aims to identify where the current dynamics of the cooperative and solidarity sector is located. To achieve this objective, experts are consulted on the scenario in which this sector is currently transiting, reviewing the fulfillment or not of the strategic objectives set out in the work entitled "Application of the strategic prospective model to the Colombian cooperative sector. Horizon: year 2031 "published in 2014 by the Institute of Studies of the Public Ministry. Similarly, evidence was sought in documents published by state agencies and the sector itself. The main finding of this work is related to the location of the sector in a similar state to that of five years ago, with some important advances, but not enough to consider transformations that lead to different scenarios of greater incidence in the life of the regions in which it develops. The foregoing leads us to conclude that if this state of affairs in the sector continues, its development will be influenced by the force of external dynamics, either from social movements or the instrumentalization of the current rulers, as has been observed. historically.

Keywords: Solidarity Economy; Cooperativism; Prospective Planning; State agencies, prospective scenario.

P13 Cooperative companies

R58 Regional development policy and planning

P21 Planning, coordination and reform

\section{Resumo}

Este artigo tem como objetivo identificar onde se situa a atual dinâmica do setor cooperativo e solidário. Para alcançar este objetivo, especialistas são consultados sobre o cenário em que este setor está atualmente transitando, analisando o cumprimento ou não dos objetivos estratégicos definidos no trabalho intitulado "Aplicação do modelo prospectivo estratégico ao setor cooperativo colombiano. Horizon: year 2031 "publicado em 2014 pelo Instituto de Estudos do Ministério Público. Da mesma forma, foram buscadas evidências em documentos publicados por órgãos estaduais e pelo próprio setor. El principal hallazgo de este trabajo se relaciona con la ubicación del sector en un estado similar al de hace cinco años, con algunos avances importantes, pero no suficientes para considerar transformaciones que lleven a escenarios distintos de mayor 
incidencia en la vida de las regiones en que se desenvolve. 0 exposto nos leva a concluir que se esse estado de coisas no setor continuar, seu desenvolvimento será influenciado pela força de dinâmicas externas, seja dos movimentos sociais, seja da instrumentalização dos atuais governantes, como se tem observado historicamente.

Palavras-chave: Economia Solidária; Cooperativismo; Planejamento Prospectivo; Órgãos estaduais, cenário prospectivo.

P13 Cooperativas

R58 Política e planejamento de desenvolvimento regional

P21 Planejamento, coordenação e reforma

\section{Introducción}

Con este artículo se pretende identificar dónde se ubica la dinámica actual del sector cooperativo y solidario, a partir de la revisión de los avances o retrocesos que se han dado para el logro de los objetivos que permiten establecer el escenario actual del sector, de acuerdo con el estudio de Obregón-Puyana et al. (2014).

Este documento presenta, inicialmente, los antecedentes que dieron origen a este trabajo; posteriormente, indica la metodología que se llevó a cabo para el logro del objetivo planteado, haciendo énfasis en el perfil de los expertos consultados y la forma en que se hizo el levantamiento de la información; luego, muestra, los hallazgos encontrados por cada variable estratégica y el escenario que enmarca la acción del sector cooperativo; en seguida, se plantea la discusión a partir de los hechos que se observan y que demuestran no haber logrado el escenario apuesta, por cada variable estratégica; finalmente, se presentan unas conclusiones.

Como aporte del artículo, se ratifican las variables estratégicas definidas en el documento de Obregón-Puyana et al. (2014), que pueden ser extensivas a las demás organizaciones solidarias; de igual manera, se indica cómo el escenario por el que transita la economía solidaria está todavía muy alejado del escenario apuesta y se sugieren acciones que permitan un acercamiento al mismo.

\section{Antecedentes}

El sector de la economía social' está en capacidad de hacer transformaciones en el rumbo actual de la economía, enfrentando los grandes problemas de concentración de riqueza y de asignación de recursos, con organizaciones que tienen como fin el

1 La Economía Social es una rama de conocimiento específico que posee su propio objeto de estudio (Rosa, 2016). 
ser humano y asumen al capital como un medio. Son muchos los estudios que así lo demuestran, tal es el caso de Serna Gómez \& Rodríguez Barrero (2016), que se muestran cómo el sector solidario es la mejor alternativa para generar desarrollo social, sostenible e inclusivo en Colombia, ante el escenario del posconflicto.

En este mismo sentido, se han elaborado varios estudios de futuro alrededor de la organización solidaria, por citar algunos: Análisis estratégico y prospectivo del sector solidario del municipio de Ocaña, hacia el año 2034 (Álvarez Arévalo y Benavides Carrascal, 2018) y el estudio Estado del arte Estudio prospectivo estratégico para el sector solidario en el departamento del Meta hacia el año 2022 (Peralta Quevedo y Herrera Niño, 2012). En el 2014, el Instituto de Estudios del Ministerio Público (IEMP), publicó un documento titulado Aplicación del modelo prospectivo estratégico al sector cooperativo colombiano, horizonte 2031 (Obregón-Puyana et al., 2014), en el que se plantea la construcción de futuro para el sector cooperativo con un horizonte que va hasta el 2031, siguiendo el modelo de planeación prospectiva².

De otra parte, es importante señalar que, en el tema de los estudios de futuro, se encuentran varias formas de discurso teórico-práctico, por lo menos seis, según Decouflé (citado por Mera Rodríguez, 2014). Mojica (citado por Obregón-Puyana et al., 2014) destaca dos corrientes que considera importantes: la corriente de la previsión, que pretende ver anticipadamente el futuro con base en el "análisis de tendencias" siempre y cuando no haya un cambio profundo o ruptura violenta del continuo de estas tendencias, ya que se fundamenta en estudios econométricos basados en la regresión estadística y uso de variables cuantificables. La otra corriente es la prospectiva, que contempla la existencia de "múltiples futuros", "considera relaciones dinámicas, incorpora variables cuantificables o no, concibe el futuro como posibilidad múltiple y lo hace depender de la acción en el presente, es decir, busca que el hombre se adueñe de su destino" (Obregón-Puyana et al., 2014, p. 19).

Es pertinente señalar que estos estudios aparecen a inicios de la década de los años 70, pero solo hasta las décadas de los 80 y 90 la academia, los gobiernos y las empresas se interesan en ellos (Henao, 2015), buscando reforzar los estudios de largo plazo y el diseño de estrategias (Bitar, 2015). En América Latina, el avance ha

2 El estudio de Obregón-Puyana et al. (2014) se toma de base para la realización de este trabajo, que hace parte de una línea de investigación emprendida por el IEMP, en la que se busca la realización de investigaciones básicas para el reconocimiento de la problemática que circunda a la economía social y solidaria, en especial a la cooperativa. En particular, este trabajo se desarrolla con la participación de expertos del sector de la economía solidaria con una visión amplia del sector, tanto por sus labores profesionales (en la academia, en el sector público y privado con relación directa en la economía social y solidaria), como por el tiempo de dedicación a este sector de la economía. Todo ello, hace que este trabajo sea considerado un referente importante de consulta sobre la hoja de ruta actual del sector cooperativo y solidario colombiano. 
sido escaso. En Colombia, en particular, se están haciendo estudios, lo que no quiere decir que se evidencie "un cambio de mentalidad hacia lo que está por venir y la necesidad de anticiparnos a ese futuro para adquirir ventajas estratégicas o una capacidad colaborativa de construir futuros deseados sino puede ser una simple obligación a la que hay que dar cumplimiento" (Rodero, 2015, p. 130).

Es así como para el desarrollo del estudio del IEMP, sus autores optaron por el modelo prospectivo en el marco de dos de las escuelas de trabajo existentes en el tema: la escuela francesa (modelo de Godet (1999)) y la colombiana (técnica de escenario apuesta propuesta por Mojica y otros antecedentes). El objetivo de este estudio es servir como orientador para el desarrollo del sector cooperativo y solidario. En la tabla 1 se presentan las cinco hipótesis consideradas (las variables estratégicas), las estrategias, objetivos y metas a lograr, y las acciones a desarrollar para alcanzar este escenario apuesta.

Tabla 1. Variables estratégicas, estrategias y objetivos/ metas

\begin{tabular}{|c|c|c|}
\hline $\begin{array}{l}\text { La variable } \\
\text { estratégica }\end{array}$ & Las estrategias & El objetivo/ la meta \\
\hline \multirow{3}{*}{$\begin{array}{l}\text { Incidencia en las } \\
\text { políticas públicas }\end{array}$} & 1. Consenso del sector & \multirow{2}{*}{$\begin{array}{l}\text { 1. Lograr un consenso en el sector cooperativo } \\
\text { y solidario frente a las políticas públicas que } \\
\text { propendan a su fortalecimiento. }\end{array}$} \\
\hline & $\begin{array}{l}\text { 2. Ministerio de la economía } \\
\text { solidario }\end{array}$ & \\
\hline & & $\begin{array}{l}\text { 2. Lograr la creación del Ministerio de la Econo- } \\
\text { mía Social y Solidaria. }\end{array}$ \\
\hline \multirow[t]{3}{*}{$\begin{array}{l}\text { Gestión gremial de la } \\
\text { Integración }\end{array}$} & $\begin{array}{l}\text { 1. Red Cooperativa con incidencia } \\
\text { nacional }\end{array}$ & \multirow{2}{*}{$\begin{array}{l}\text { 1. Lograr que el 90\% cooperativas y OS locales } \\
\text { conformen red nacional de intercoopera- } \\
\text { ción. }\end{array}$} \\
\hline & 2. Cooperativa compra coope- & \\
\hline & rativa & $\begin{array}{l}\text { 2. Lograr que el } 50 \% \text { cooperativas y OS deman- } \\
\text { de } 75 \% \text { de productos y servicios ofrecidos } \\
\text { por el mismo sector solidario y cooperativo. }\end{array}$ \\
\hline \multirow[t]{4}{*}{$\begin{array}{l}\text { Identidad solidaria y } \\
\text { de cooperación }\end{array}$} & $\begin{array}{l}\text { 1. Usos de los servicios coope- } \\
\text { rativos }\end{array}$ & $\begin{array}{l}\text { 1. Lograr que más del } 90 \% \text { de los asociados } \\
\text { usen los servicios de las cooperativas. }\end{array}$ \\
\hline & 2. Asamblea General de asociados & \multirow{2}{*}{$\begin{array}{l}\text { 2. Lograr que la participación en las Asambleas } \\
\text { Generales supere el } 90 \% \text { de los asociados. }\end{array}$} \\
\hline & \multirow[t]{2}{*}{ 3. Asamblea de delegados } & \\
\hline & & $\begin{array}{l}\text { 3. Lograr que las cooperativas que convocan } \\
\text { asambleas de delegados tengan la capaci- } \\
\text { dad de realimentarse con los asociados de } \\
\text { base. }\end{array}$ \\
\hline Vínculo Asociativo & El balance social & $\begin{array}{l}\text { Lograr que el } 90 \% \text { de las cooperativas y OS } \\
\text { orienten su gestión }\end{array}$ \\
\hline \multirow[t]{2}{*}{$\begin{array}{l}\text { Investigación sobre el } \\
\text { modelo cooperativo }\end{array}$} & \multirow[t]{2}{*}{ Grupos y líneas de investigación } & $\begin{array}{l}\text { 1. Lograr que más del } 90 \% \text { de las universidades } \\
\text { tengan grupos de investigación con líneas } \\
\text { sobre Economía Solidaria respaldadas por } \\
\text { la política nacional de Ciencia, Tecnología e } \\
\text { Innovación, con impacto en el sector. }\end{array}$ \\
\hline & & $\begin{array}{l}\text { 2. Lograr que los centros e institutos indepen- } \\
\text { dientes cuenten con líneas de investigación } \\
\text { afines a la temática con impacto en el sector. }\end{array}$ \\
\hline
\end{tabular}


El principal resultado del estudio de Obregón-Puyana et al. (2014) fue identificar posibles escenarios, dado el cumplimiento o no de los eventos planteados resultantes de la metodología aplicada. En el núcleo tendencial, conformado por los escenarios de mayor probabilidad de ocurrencia, se incluyó el escenario apuesta, o escenario deseado, como norte a lograr. En el mismo documento se sugiere un camino para alcanzarlo y las estrategias a seguir. A ese escenario se le denominó "nuevo modelo socioeconómico" al que se espera llegar en 2031, cuando se cumplen cien años de la aparición de la ley cooperativa en el país. Este escenario implica un exigente reto, pues supone el pleno alcance de las cinco estrategias observadas en la tabla 1. El no cumplimiento de alguna de ellas nos lleva a escenarios distintos.

Con este artículo se pretende responder la pregunta: ¿dónde se ubica la dinámica actual del sector cooperativo y solidario? Para resolver este interrogante se revisan los avances o retrocesos que se han dado en la dinámica del sector que inciden en el logro de los objetivos estratégicos planteados en la tabla 1 para, de esta forma, establecer el escenario en que se ubica el sector de acuerdo con Obregón-Puyana et al. (2014).

\section{Material y método}

El artículo se basa en una investigación de tipo cualitativo, en el cual se consultaron 23 expertos, conocedores del sector cooperativo y solidario, para que dieran a conocer su percepción sobre el estado actual del sector en cada una de las variables estratégicas analizadas. Para identificar el escenario en el que se ubica el sector actualmente (según los escenarios previstos en el documento del IEMP), y para analizar las líneas de acción a futuro, se realizó un taller con un grupo focal conformado por 14 expertos.

\section{Perfil de los expertos consultados}

De los 23 expertos consultados, el 83\% tiene más de 10 años de conocimiento del sector cooperativo y solidario, bien sea porque son estudiosos del tema o porque han ejercido funciones en el sector público o privado en relación con el sector; el 9\% tiene más de 5 años de conocimiento del sector similar al indicado anteriormente; y con el $9 \%$ restante no se pudo identificar el tiempo de conocimiento del sector. Todos los expertos son profesionales, el 87\% tiene formación posgradual, el 13\% restante no la tiene, pero son estudiosos del tema, con muchos años de experiencia. De los 20 expertos que tienen formación posgradual, en curso o terminada, el 5\% tiene 
formación postdoctoral, el 30\% de doctorado, el 50\% de maestría y el 15\% restante de especialización.

El 65\% de los consultados actualmente está vinculado a la academia y el 35\% restante está directamente vinculado con organizaciones del sector. Sin embargo, independientemente del sector en que se encuentren ubicados actualmente, se observa que ha habido migración de académicos al sector o del sector a la academia. Lo que les da una visión holística. Hay que advertir que la respuesta a las preguntas del cuestionario puede ser objeto de sesgo, por las siguientes circunstancias:

- El $30 \%$ de los consultados son mujeres y el 70\% restante son hombres, esperamos que el sesgo se atenúe por la diversidad en la formación académica y por la experiencia misma en el sector.

- Todos los expertos consultados tienen formación en ciencias sociales. Sin embargo, el 77\% tienen profesiones relacionadas directamente con las organizaciones, tales como economía (27\%), administración de empresas (19\%), ingeniería industrial (15\%), contaduría pública (12\%) y derecho (4\%). Se espera que este sesgo sea contrarrestado por las posiciones del 23\% restante, que tienen profesiones relacionadas con las humanidades y no directamente con las organizaciones, educación (8\%), sociología (4\%), ecología (4\%), licenciatura en filosofía (4\%) y autodidacta en las ciencias sociales (4\%).

Todas estas cifras dan garantía de que los expertos consultados tienen una visión amplia del sector y sus opiniones son calificadas para tomarle el pulso.

\section{Levantamiento de información}

Para el levantamiento de información, se aplicó un cuestionario a expertos seleccionados de acuerdo con el perfil indicado anteriormente, se realizó un grupo focal y se consultaron sobre documentos producidos por entidades públicas y privadas relacionadas directamente con el sector. 


\section{Resultados}

\section{Dinámica actual del sector cooperativo y solidario: avances y retrocesos}

El cuestionario tuvo como finalidad obtener información sobre la percepción de los expertos en relación con la dinámica de las organizaciones solidarias que tiene que ver con las hipótesis planteadas en el documento de Obregón-Puyana et al. (2014).

Para la exposición de los hallazgos se desarrolla el contenido de la tabla 1. Se tiene en cuenta, primero, la definición para cada variable estratégica en el documento del IEMP, luego, se indican las estrategias planteadas en el mismo documento con sus respectivos objetivos para lograr el escenario apuesta; posteriormente, se expone lo indicado por los expertos consultados en cuanto a los avances o retrocesos para cada objetivo (este indicativo se hace separando a los expertos vinculados a la academia de los expertos vinculados al sector); por último, se despliegan las acciones observadas por los expertos consultados.

\section{Variable estratégica: Incidencia en las políticas públicas}

Se define como la capacidad de influir de forma directa en el ciclo de las políticas públicas generales y sectoriales que inciden en el sector cooperativo. Para lograr incidencia en política pública se consideraron dos estrategias en el trabajo del IEMP: Consenso del sector y la creación del Ministerio de Economía Solidaria.

\section{Estrategia 1: Consenso del sector}

Objetivo/meta: lograr un consenso en el sector cooperativo y solidario frente a las políticas públicas que propendan a su fortalecimiento.

Tabla 2. Percepción consensos regionales

\begin{tabular}{llccccc}
\hline & \multicolumn{2}{c}{ Sector } & \multicolumn{2}{c}{ Academia } & \multicolumn{2}{c}{ Total } \\
\hline Avances & 2 & $25 \%$ & 8 & $53 \%$ & 10 & $43 \%$ \\
\hline Retrocesos & 2 & $25 \%$ & 1 & $7 \%$ & 3 & $13 \%$ \\
\hline Ambos & 2 & $25 \%$ & 2 & $13 \%$ & 4 & $17 \%$ \\
\hline No ha habido & 1 & $13 \%$ & 4 & $27 \%$ & 5 & $22 \%$ \\
\hline No sabe nor & 1 & $13 \%$ & & $0 \%$ & 1 & $4 \%$ \\
\hline & 8 & $100 \%$ & 15 & $100 \%$ & 23 & $100 \%$ \\
\hline
\end{tabular}


La tabla 2 presenta la percepción de los consultados frente a la generación de consensos regionales, como dato curioso, se puede observar que quienes están vinculados al sector son más críticos frente a logro de este objetivo, el 52\% de los académicos reconocen mayores avances en este sentido frente al 25\% de los vinculados al sector; el $7 \%$ de los académicos reconocen retrocesos frente al $25 \%$ de los vinculados al sector, que reconocen que hay retrocesos; el 13\% de los vinculados al sector indican que no saben, no responden, frente a los académicos; que ninguno señale que no sabe, no responde, quiere decir que tienen mayor grado de certeza. Valdría la pena analizar si la imagen que proyecta el sector está mostrando mayores niveles de concertación que en el pasado, pero que aún no es suficiente para lograr consensos que contribuyan con el fortalecimiento del sector. Las acciones para el cumplimiento de esta estrategia, observadas en el 2019 por los expertos consultados, se resumen a continuación.

Los expertos mencionaron que se han presentado ciertos avances producto de consensos regionales para formulación de política pública con algunos gobiernos departamentales y locales, siendo el caso de mayor impacto, lo logrado en el departamento de Antioquia y la ciudad de Medellín, aunque se mencionaron los realizados en Cali, el departamento del Huila, Bogotá y la región Central, teniendo mayor o menor incidencia, dependiendo de la voluntad de los gobiernos locales y departamentales, con resultados que no son claros ni determinantes, tanto por la falta de voluntad política del Estado en sus diferentes niveles como por la carencia de una fuerza motivadora por parte del sector, ya que los esfuerzos realizados por algunas entidades de integración del sector representan a las organizaciones solidarias reconocidas que prestan servicios de ahorro y crédito.

También se mencionaron acuerdos logrados por las agremiaciones como Analfe, Fecolfin y Ascoop para tener un solo discurso frente a organismos públicos como la Dian o la Superintendencia de la Economía Solidaria. Otros avances mencionados son los siguientes: los procesos realizados por Redess y la Mesa Nacional de Economía Solidaria y Social; el proceso que generó el Acuerdo de Paz, en que se rescata el debate alrededor del Planfes presentado por la UAEOS; el debate sobre el Plan Nacional de Desarrollo, que dio origen al "Pacto por la economía cooperativa y solidaria - Confecoop, así como la firma de pactos por la Paz y la ESS por parte de algunos actuales Congresistas y el hoy Presidente de la República. El aspecto más preocupante ha sido el retroceso en cuanto al papel que el actual gobierno está dando al acuerdo de paz.

Frente a las acciones que proponía el estudio realizado por el IEMP, se puede comentar que no se han tenido en cuenta, quedando pendiente su análisis y posible 
realización por parte del sector. La actividad más cercana fueron las realizadas para ofrecer un aporte al plan nacional de desarrollo

\section{Estrategia 2: Creación Ministerio de Economía Social y Solidaria}

Objetivo/meta: lograr la creación del Ministerio de la Economía Social y Solidaria.

Tabla 3. Percepción de acciones encaminadas a la creación del Ministerio de la Economía Solidaria

\begin{tabular}{lcccccc}
\hline & \multicolumn{2}{c}{ Sector } & \multicolumn{2}{c}{ Academia } & \multicolumn{2}{c}{ Total } \\
\hline Avances & 1 & $13 \%$ & 1 & $7 \%$ & 2 & $9 \%$ \\
\hline Retrocesos & 2 & $25 \%$ & 4 & $27 \%$ & 6 & $26 \%$ \\
\hline Ambos & 0 & $0 \%$ & & $0 \%$ & & $0 \%$ \\
\hline No ha habido & 4 & $50 \%$ & 8 & $53 \%$ & 12 & $52 \%$ \\
\hline No sabe nor & 1 & $13 \%$ & 2 & $13 \%$ & 3 & $13 \%$ \\
\hline & 8 & $100 \%$ & 15 & $100 \%$ & 23 & $100 \%$ \\
\hline
\end{tabular}

La tabla 3 muestra la percepción de los consultados frente a las acciones para el cumplimiento de esta estrategia. Allí se observa que el 52\% de los consultados reconocen un estancamiento en el sentido de procurar la conformación de un Ministerio de la Economía Solidaria que sirva de dinamizador de la política pública que contribuya al fortalecimiento del sector; el 26\% reconocen que ha habido un retroceso en la búsqueda de la conformación del Ministerio de la Economía Solidaria. Lo anterior indica que el 78\% de los consultados consideran que la creación del Ministerio no ha sido una prioridad. En ello hay más o menos una percepción similar tanto de actores vinculados actualmente al sector como de los actores vinculados a la academia. Las acciones para el cumplimiento de esta estrategia, observadas en el 2019 por los expertos consultados, se resumen de a continuación.

Los expertos consideraron que no hay avances significativos y que se han presentado situaciones de reducida significancia como han sido las conversaciones entre la Mesa nacional, Confecoop, Analfe y la UAEOS. Para los expertos ha habido más bien retrocesos significativos como el hecho de pasar de un Departamento Nacional (Dansocial) a una Unidad Administrativa (UAEOS) adscrita al Ministerio de trabajo, situación que refleja que no se ha tenido la suficiente incidencia política para motivar e impulsar la idea del Ministerio para el sector, idea que no "tiene el consenso en el sector, ni sensibilización al interior del gobierno, ni ambiente político en el congreso ni el apoyo de la base social". Es decir, "no hay condiciones objetivas" para impulsar esta propuesta. Frente a las acciones que se propusieron en el estudio, la que ha avanzado 
ha sido la de consolidar un grupo de congresistas y los esfuerzos para continuar con la idea de realizar mediciones del impacto que la economía solidaria y social tiene en el país.

Las acciones que procuraran mayor incidencia en política pública más importantes mencionadas por los expertos fueron las siguientes: a nivel de política pública regional, las ordenanzas departamentales de Antioquia y Santander, el acuerdo municipal de Medellín y acciones de incidencia en Huila, así como la participación de la Red Unicossol en el Consejo Nacional de Planeación con dos representantes, uno por el sector social y el sector educativo. A nivel de política nacional, la inclusión en el Plan nacional de desarrollo de algunos artículos para fortalecer al sector cooperativo y solidario

Desde el sector, se mencionaron los encuentros y conversatorios nacionales y regionales promovidos por la Convergencia alternativa social y solidaria por la paz, la Mesa Nacional de Economía Social y Solidaria, así como varios grupos focales que llevó a cabo Analfe en distintas ciudades del país, para conocer los aspectos en que los Fondos tienen más incidencia y la propuesta programática de la Mesa Nacional de ESS para candidatos a Alcaldías y gobernaciones. También se mencionaron los pactos de instituciones internacionales con aportes de las entidades de integración del sector cooperativo, mutualista y del sector fondista Confecoop, Analfe para impulsar una "presión internacional" que favorezca la creación de política pública en los diferentes Estados y gobiernos de la región latinoamericana.

Desde la academia, se mencionaron las reuniones para promover el currículo global del cooperativismo y la economía solidaria, los enlaces nacionales sobre "la paz y la otra economía con solidaridad es posible" y la Cátedra libre promovida por la Universidad Cooperativa de Colombia y apoyada por la Red Unicossol con base en un proyecto multicampus de la ucc para conocer e integrar los avances en políticas públicas en siete campus en Colombia, así como la participación de los investigadores en proyectos orientados a mostrar la importancia de las organizaciones solidarias y la socialización de los resultados ante instancias académicas y gubernamentales.

\section{Variable estratégica: Gestión gremial de la integración}

La integración de organizaciones, tanto horizontal como verticalmente, favorece las economías de escala, así como la articulación como gremio económico-político, que favorece la comprensión de temáticas comunes. Los estilos de agremiación política y económica deben ser complementarios, considerando la importancia de la base social en la toma de decisiones. 
Para establecer la gestión gremial se consideraron dos estrategias en el trabajo del IEMP: 1) red cooperativa con incidencia nacional e internacional y 2) cooperativa compra cooperativa. Los objetivos propuestos en ese trabajo, así como las acciones observadas en la actualidad por los expertos consultados, se indican a continuación.

\section{Estrategia 3: Red cooperativa con incidencia nacional e internacional}

Objetivo/meta: lograr que el 90\% de las cooperativas locales conformen una red nacional de 'intercooperación'.

Tabla 4. Red nacional de intercooperación

\begin{tabular}{lcccccc}
\hline & \multicolumn{2}{c}{ Sector } & \multicolumn{2}{c}{ Academia } & \multicolumn{2}{c}{ Total } \\
\hline Avances & 3 & $38 \%$ & 5 & $33 \%$ & 8 & $35 \%$ \\
\hline Retrocesos & 1 & $13 \%$ & 2 & $13 \%$ & 3 & $13 \%$ \\
\hline Ambos & 3 & $38 \%$ & 3 & $20 \%$ & 6 & $26 \%$ \\
\hline No ha habido ni avance & 1 & $13 \%$ & 3 & $20 \%$ & 4 & $17 \%$ \\
\hline No sabe no responde & 0 & $0 \%$ & 2 & $13 \%$ & 2 & $9 \%$ \\
\hline & 8 & $100 \%$ & 15 & $100 \%$ & 23 & $100 \%$ \\
\hline
\end{tabular}

La tabla anterior muestra la percepción de los consultados frente a acciones que fortalezcan una red nacional de intercooperación. Se observa que el 35\% de los consultados considera que se han dado avances en la conformación de la mencionada red, siendo más optimistas los vinculados al sector (38\% del total del sector); el $26 \%$ considera que ha habido avances y retrocesos al mismo tiempo (en este caso, la percepción más alta es la de representantes del sector). Estas cifras pueden reflejar que a pesar de que existan retrocesos y a veces estancamiento, existe una percepción positiva para la conformación de la red. A continuación, se presentan las acciones observadas actualmente por los expertos en la conformación de la "red nacional de intercooperación".

Los expertos provenientes del sector mencionaron nuevamente la Mesa Nacional de ESS y las Mesas Regionales de economía social y solidaria por la paz, los nodos de la Convergencia alternativa social y solidaria por la paz y los Encuentros nacionales de jóvenes y mujeres solidarios como intentos de "convocar voluntades de los cooperativistas, mutualistas, comunitaristas, fondos de empleados, organizaciones sociales para actuar como un gran bloque nacional solidario" y el trabajo con redes de jóvenes (Cincop), así como la existencia de diferentes tipos de redes de carácter regional mas no nacional, como las redes de servicios financieros Coopcentral 
- Visionamos, la Red servicios financieros Conecta, Red de intercooperación en Antioquia (Seguros y compras comunes).

Sin embargo, se considera que estos esfuerzos han sido insuficientes, dispersos y centrados en algunas organizaciones solidarias de importancia, pero provenientes de diferentes corrientes de pensamiento, demostrando una debilidad de integración intergremial, que refleja poco interés en adelantar acciones conjuntas en procura de propósitos más elevados y comprometidos para el bien del sector. También, se mencionó, desde la academia, la consolidación de la Red Unicossol y la creación de la red Ciriec Colombia que, en conjunto, agrupan más de 50 profesores.

En relación con las acciones previstas por el estudio se han producido solo intentos de promoción de ferias locales, regionales y nacionales con organizaciones solidarias, pero sin avances significativos en la idea de consolidar un sello de origen productivo solidario, que sigue siendo una interesante propuesta sin mayor desarrollo, contándose con un impulso que Agrosolidaria viene realizando con este sentido.

\section{Estrategia 4: Cooperativa compra cooperativa}

Objetivo/meta: lograr que el 50\% de las cooperativas demande el $75 \%$ de productos y servicios ofrecidos por el mismo sector.

Tabla 5. Cooperativa compra cooperativa

\begin{tabular}{lcccccc}
\hline & \multicolumn{2}{c}{ Sector } & \multicolumn{2}{c}{ Academia } & \multicolumn{2}{c}{ Total } \\
\hline Avances & 0 & $0 \%$ & 5 & $33 \%$ & 5 & $22 \%$ \\
\hline Retrocesos & 0 & $0 \%$ & 0 & $0 \%$ & 0 & $0 \%$ \\
\hline Ambos & 3 & $38 \%$ & 3 & $20 \%$ & 6 & $26 \%$ \\
\hline No ha habido ni avance & 4 & $50 \%$ & 5 & $33 \%$ & 9 & $39 \%$ \\
\hline No sabe no responde & 1 & $13 \%$ & 2 & $13 \%$ & 3 & $13 \%$ \\
\hline & 8 & $100 \%$ & 15 & $100 \%$ & 23 & $100 \%$ \\
\hline
\end{tabular}

La tabla anterior muestra que los expertos vinculados al sector actualmente observan un estancamiento en el desarrollo de este objetivo, el 50\% de los consultados indican que no ha habido ni avance ni retroceso, el 38\% indica que existen acciones de avance y retroceso y el 12\% no sabe no responde. Entre tanto, los representantes de la academia son un poco más optimistas, el 33\% de los consultados indica que ha habido avances, el 20\% que ha habido tanto avances como retrocesos, el 33\% no ha habido ni avances ni retroceso, 13\% no sabe no responde. A continuación, se presentan las acciones observadas en el 2019 por los expertos consultados en el sentido de procurar la estrategia. 
Los expertos mencionaron la existencia de algunas acciones como ferias, eventos de promoción de emprendedores solidarios, utilización de emprendimientos solidarios, en eventos del sector que son un síntoma de que se están "sembrado semillas de integración". Ejemplos: Agrosolidaria, la articulación de aseguramiento en materia de riesgos, comunicación y mercadeo en los servicios de ahorro y crédito. sin embargo, se consideran experiencias muy locales todavía sin una proyección nacional.

En cuanto a las acciones que proponía el estudio se ha hecho muy poco. A las ya mencionadas anteriormente por los expertos, se añadieron unas acciones de carácter muy puntual como: la subcomisión de Balance Social en la Superintendencia de la Economía solidaria (Confecoop, Analfe), la consolidación de una red de organizaciones solidarias de salud, la $\vee$ semana internacional de la economía social y solidaria impulsada por Unicossol, la propuesta de Cooperativas por Colombia de Confecoop. Estas acciones se consideran más como esfuerzos fragmentados, que no tienen la capacidad de liderar procesos de integración de mayor peso a nivel nacional.

\section{Variable estratégica: Identidad solidaria y de cooperación}

Lo característico del sistema de economía solidaria: cultura, educación, principios, valores (transparencia), coherencia entre el pensar y el actuar. Actitud de los individuos frente a las demás personas y las cosas desde la gestión democrática de la organización y la ayuda mutua.

\section{Estrategia 5: Usos de servicios cooperativos}

Objetivo/meta: lograr que más del 90\% de los asociados haga uso de los servicios de su cooperativa.

Tabla 6. Uso de los servicios de la organización solidaria por parte de los asociados

\begin{tabular}{lcccccc}
\hline & \multicolumn{2}{c}{ Sector } & \multicolumn{2}{c}{ Academia } & \multicolumn{2}{c}{ Total } \\
\hline Avances & 4 & $50 \%$ & 4 & $27 \%$ & 8 & $35 \%$ \\
\hline Retrocesos & 3 & $38 \%$ & 2 & $13 \%$ & 5 & $22 \%$ \\
\hline Ambos & 1 & $13 \%$ & 3 & $20 \%$ & 4 & $17 \%$ \\
\hline No ha habido ni avances ni retrocesos & 0 & $0 \%$ & 4 & $27 \%$ & 4 & $17 \%$ \\
\hline No sabe no responde & 0 & $0 \%$ & 2 & $13 \%$ & 2 & $9 \%$ \\
\hline & 8 & $100 \%$ & 15 & $100 \%$ & 23 & $100 \%$ \\
\hline
\end{tabular}


La anterior tabla muestra que el 35\% de los consultados perciben que ha habido avances en acciones que promueven el uso de los servicios de las organizaciones solidarias por parte de los asociados. Se destaca la percepción de los representantes del sector, el 50\% de ellos consideran que hay avances. El 22\% de los consultados perciben que ha habido retrocesos en acciones que estimulen el uso de los servicios de la organización solidaria por parte de los asociados, se destaca también la percepción de los vinculados al sector, el $38 \%$ de ellos considera que ha habido retrocesos en este sentido. Por último, se resalta que el $27 \%$ y el $13 \%$ de los vinculados a la academia perciben, respectivamente, que no ha habido ni avances ni retrocesos y que no saben no responden. A continuación, se presentan las acciones observadas en el 2019 por los expertos consultados en el uso de los servicios de las organizaciones solidarias por parte de los asociados.

En el caso de algunas de las organizaciones solidarias que prestan servicios de ahorro y crédito, apenas se están comenzando a utilizar las nuevas tecnologías para ofrecer los servicios financieros. En esta actividad hay organizaciones ofreciendo precios justos, tanto para el crédito como para el ahorro, lo que marca una diferencia con la banca comercial. En otros casos el asociado identifica a su organización más como un banco y otros prefieren usar los productos de la banca comercial. Se evidencia la necesidad de crear nuevos modelos de mercadeo por parte de todas las empresas del sector. En el caso de los servicios educativos, para las cooperativas se valora que el Estado ha fijado la destinación de los recursos asignados vía la distribución del excedente. Al tener en cuenta las acciones propuestas en el estudio, se evidencia que no se han desarrollado, unas porque el sector no las propone y, en el caso de la educación cooperativa, porque el Estado se ha apoderado de estos recursos.

\section{Estrategia 6: Asamblea general de asociados}

Objetivo/meta: lograr que la participación en las asambleas generales supere el 90\% de los asociados.

Tabla 7. Avances o retrocesos en la participación en las asambleas generales

\begin{tabular}{lcccccc}
\hline & \multicolumn{2}{c}{ Sector } & \multicolumn{2}{c}{ Academia } & \multicolumn{2}{c}{ Total } \\
\hline Avances & 2 & $25 \%$ & 2 & $13 \%$ & 4 & $17 \%$ \\
\hline Retrocesos & 3 & $38 \%$ & 6 & $40 \%$ & 9 & $39 \%$ \\
\hline Ambos & 2 & $25 \%$ & 1 & $7 \%$ & 3 & $13 \%$ \\
\hline No ha habido ni avances ni retrocesos & 1 & $13 \%$ & 2 & $13 \%$ & 3 & $13 \%$ \\
\hline No sabe no responde & 0 & $0 \%$ & 4 & $27 \%$ & 4 & $17 \%$ \\
\hline & 8 & $100 \%$ & 15 & $100 \%$ & 23 & $100 \%$ \\
\hline
\end{tabular}


En la tabla 7 se puede apreciar que el $39 \%$ de los consultados consideran que hay retrocesos en lo relacionado con la participación en las asambleas generales. El $17 \%$ de las respuestas considera que hubo avances, siendo más afirmativa la respuesta de los consultados provenientes del sector, donde el $25 \%$ considera que hubo avances. El 13\% de los consultados coinciden en considerar que ha habido tanto retrocesos como avances, a la vez que no se han presentado ni avances, ni retrocesos. Es interesante anotar que el $17 \%$ de los que respondieron no sabe no responden, pertenecen a la academia en su totalidad. A continuación, se presentan las acciones observadas en el 2019 por los expertos consultados en el sentido de promover la participación de los asociados en las asambleas.

Los expertos consideran que se ha incrementado el uso de la virtualidad en las asambleas para el desarrollo del proceso electoral. Sin embargo, la participación en este proceso por parte de los asociados tiende a ser reducido y son pocas las organizaciones solidarias que tienen promedios altos de participación de los asociados en este proceso. La mayoría de los asociados no comprende la gestión social, provocando un desequilibrio de la gestión económica que consolida en el asociado más su acción como usuario que como dueño en un buen número de organizaciones.

\section{Estrategia 7: Asamblea de delegados}

Objetivo/meta: lograr que las cooperativas que convocan asambleas de delegados tengan la capacidad de realimentarse con los asociados de base.

Tabla 8. Avances y retrocesos realimentación asamblea de delegados

\begin{tabular}{lcccccc}
\hline & \multicolumn{2}{c}{ Sector } & \multicolumn{2}{c}{ Academia } & \multicolumn{2}{c}{ Total } \\
\hline Avances & 1 & $13 \%$ & 2 & $13 \%$ & 3 & $13 \%$ \\
\hline Retrocesos & 3 & $38 \%$ & 8 & $53 \%$ & 11 & $48 \%$ \\
\hline Ambos & 3 & $38 \%$ & 0 & $0 \%$ & 3 & $13 \%$ \\
\hline No ha habido ni avances ni retrocesos & 1 & $13 \%$ & 1 & $7 \%$ & 2 & $9 \%$ \\
\hline No sabe no responde & 0 & $0 \%$ & 4 & $27 \%$ & 4 & $17 \%$ \\
\hline & 8 & $100 \%$ & 15 & $100 \%$ & 23 & $100 \%$ \\
\hline
\end{tabular}

El resultado de la consulta (tabla 8) muestra que el 48\% de los expertos considera que hay un retroceso en el tema de la realimentación de los delegados a sus electores de lo que ha acaecido en la asamblea, en tanto que el 13\% considera que hay avances. Otro 13\% considera que ha habido avances y retrocesos, siendo interesante anotar que las respuestas corresponden a los expertos provenientes del sector. No sabe no responde el $17 \%$ de los consultados, todos ellos pertenecientes a 
la academia. Un 9\% considera que no ha habido ni avances ni retrocesos. En resumen, se puede considerar que la mayoría de las respuestas muestran una visión pesimista respecto del proceso de realimentación de los delegados a sus bases sociales sobre los resultados de la Asamblea. A continuación, se muestran las acciones observadas en el 2019 por los expertos consultados en el sentido de procurar la realimentación en la asamblea de delegados.

En términos generales los delegados opinaron que no se desarrolla una realimentación efectiva de los delegados hacia las bases sociales de los resultados de la asamblea y son pocas las organizaciones en que los delegados tienen clara esta responsabilidad. Es necesario anotar que la asamblea de delegados es propia de las organizaciones solidarias que llegan a un nivel de crecimiento que les exige pasar de la asamblea de asociados a la de delegados.

En cuanto a las acciones previstas en el estudio se considera que no han sido objeto de atención por el sector. Los expertos consideran que se han realizado acciones educativas y campañas para fortalecer la identidad, pero, una buena fracción de asociados no desarrolla su vínculo de pertenencia como dueños y usuarios, en parte porque no son conscientes del impacto logrado por las organizaciones solidarias, ya que no hay suficientes acciones que informen sobre los logros obtenidos a través de las organizaciones del sector.

\section{Variable estratégica: Vínculo asociativo}

El vínculo asociativo tiene que ver con la relación entre el asociado y la cooperativa.

\section{Estrategia 8: Balance social}

Objetivo/meta: lograr que el 90\% de las cooperativas orienten su gestión con base en su Balance Social.

Tabla 9. Avances o retrocesos del Balance Social como orientador de la gestión en las organizaciones solidarias

\begin{tabular}{lcccccc}
\hline & \multicolumn{2}{c}{ Sector } & \multicolumn{2}{c}{ Academia } & \multicolumn{2}{c}{ Total } \\
\hline Avances & 3 & $38 \%$ & 6 & $40 \%$ & 9 & $39 \%$ \\
\hline Retrocesos & 1 & $13 \%$ & 1 & $7 \%$ & 2 & $9 \%$ \\
\hline Ambos & 4 & $50 \%$ & 3 & $20 \%$ & 7 & $30 \%$ \\
\hline No ha habido ni avances ni retrocesos & 0 & $0 \%$ & 2 & $13 \%$ & 2 & $9 \%$ \\
\hline No sabe no responde & 0 & $0 \%$ & 3 & $20 \%$ & 3 & $13 \%$ \\
\hline & 8 & $100 \%$ & 15 & $100 \%$ & 23 & $100 \%$ \\
\hline
\end{tabular}


La tabla anterior muestra que el 39\% de los consultados consideran que hay avances en el tema del balance social, en tanto que el 30\% consideró que había avances y retrocesos, por lo tanto, se puede percibir una visión favorable sobre el avance en el uso del balance social como orientador de la gestión. Un 9\% de las respuestas dadas consideró que había retrocesos y otro 9\% respondió que no había avances ni retrocesos. Un 13\% de los consultados, todos ellos de la academia, no saben o no responden. A continuación, se muestran las acciones observadas en el 2019 por los expertos consultados en el uso del balance social como orientador de la gestión en las organizaciones solidarias.

Los expertos participantes en el taller coincidieron en que la administración de las entidades solidarias, en general, desarrollan diversas acciones en el tema del balance social, haciéndolo a su manera, desde una perspectiva mayoritariamente cualitativa y con un carácter más asistencial al mostrar principalmente los beneficios que se le dan al asociado y no lo que la entidad recibe de su asociado. Los expertos de la academia consideran que se están haciendo esfuerzos para desarrollar propuestas de instrumentos que permitan hablar de un balance social y reconocen la propuesta de balance social que está preparando la Superintendencia de la economía solidaria como importante, acompañándola a través de un trabajo integrado en una mesa de trabajo.

Los expertos consultados, en relación con otras actividades de fortalecimiento del vínculo asociativo, opinan que se están desarrollando cada vez más estrategias comunicativas y educativas para fortalecer el vínculo asociativo pero los asociados parecieran no responder a los esfuerzos realizados y siguen comportándose solo como usuarios

\section{Variable estratégica: Investigación sobre el modelo cooperativo}

Construcción de conocimiento del sector cooperativo, que contribuya con su evolución y la de las personas que lo conforman.

\section{Estrategia 9: Grupos y líneas de investigación}

Objetivo/meta: lograr que más del 90\% de las universidades tengan grupos de investigación con líneas sobre Economía Solidaria respaldadas por la política nacional de Ciencia, Tecnología e Innovación, con impacto en el sector. 
Tabla 10. Avances o retrocesos de la Investigación sobre el modelo cooperativo y solidario

\begin{tabular}{lcccccc}
\hline & \multicolumn{2}{c}{ Sector } & \multicolumn{2}{c}{ Academia } & \multicolumn{2}{c}{ Total } \\
\hline Avances & 2 & $25 \%$ & 10 & $67 \%$ & 12 & $52 \%$ \\
\hline Retrocesos & 1 & $13 \%$ & & $0 \%$ & 1 & $4 \%$ \\
\hline Ambos & 3 & $38 \%$ & 3 & $20 \%$ & 6 & $26 \%$ \\
\hline No ha habido ni avances ni retrocesos & 1 & $13 \%$ & 2 & $13 \%$ & 3 & $13 \%$ \\
\hline No sabe no responde & 1 & $13 \%$ & 0 & $0 \%$ & 1 & $4 \%$ \\
\hline & 8 & $100 \%$ & 15 & $100 \%$ & 23 & $100 \%$ \\
\hline
\end{tabular}

Las respuestas dadas por los expertos consultados en relación con la promoción de grupos y centros de investigación se reflejan en la tabla anterior. Allí se puede observar que un 52\% de las respuestas hacen mención a avances, proviniendo de los expertos pertenecientes a la academia en un 67\% y un 25\% a los expertos del sector. El 26\% consideraban que había avances y retrocesos, en tanto que un 13\% de las respuestas se referían a que no había ni avances ni retrocesos. El 4\% respondió que había retrocesos, correspondiendo a una respuesta de un experto del sector. Y otro experto del sector (4\%) respondió que no sabe o no responde. A continuación, se muestran las acciones observadas en el 2019 por los expertos consultados en relación con la promoción de la investigación sobre el modelo cooperativo y solidario.

Los expertos participantes en el taller consideraron que ha crecido el número de profesores, grupos, líneas y proyectos de investigación relacionados con el tema de la economía social y solidaria y se han consolidado dos redes nacionales (Unicossol y Ciriec Colombia), así como el número de universidades interesadas en el tema. Sin embargo, vale señalar que la economía social y solidaria todavía no es ampliamente aceptada por la comunidad científica tradicional.

Un hecho importante fueron los seis estudios realizados por el Instituto de Estudios del Ministerio Público en el periodo 2010-2016 alrededor del sector cooperativo, en los cuales hubo una activa participación de los profesores de la red Unicossol y Ciriec-Colombia, así como la existencia del observatorio de Fondos de Empleados creado por Analfe. Las redes mencionadas realizan anualmente una serie de eventos para difundir los resultados de los proyectos de investigación. Los procesos de acreditación que exige el Ministerio de Educación tienen una influencia negativa para la consolidación de asignaturas en los programas de pre y posgrado, pues consideran de poca utilidad científica la existencia de cursos y asignaturas sobre el tema. Un aspecto importante es la aparición de Doctores y doctorandos en los temas relacionados con la economía solidaria y social. También se lamenta la desaparición de los 
centros de investigación impulsados por el gremio (Cenicoop) y el de una entidad del sector, así como la disminución de programas de pre y posgrado dedicados al tema.

En relación con las dos primeras acciones recomendadas por el estudio de prospectiva poco o nada se ha logrado. En la tercera actividad propuesta sí ha habido un incremento en el fomento y fortalecimiento de redes de investigadores con diferentes mecanismos (pasantías de investigación, semilleros de investigación).

\section{Estrategia 10: Centros independientes de investigación}

Objetivo/meta: lograr que los centros e institutos independientes cuenten con líneas de investigación afines a la temática con impacto en el sector.

Tabla 11. Avances o retrocesos de la Investigación centros independientes de investigación

\begin{tabular}{lcccccc}
\hline & \multicolumn{2}{c}{ Sector } & \multicolumn{2}{c}{ Academia } & \multicolumn{2}{c}{ Total } \\
\hline Avances & 0 & $0 \%$ & 3 & $20 \%$ & 3 & $13 \%$ \\
\hline Retrocesos & 1 & $13 \%$ & 4 & $27 \%$ & 5 & $22 \%$ \\
\hline Ambos & 2 & $25 \%$ & 4 & $27 \%$ & 6 & $26 \%$ \\
\hline No ha habido ni avances ni retrocesos & 4 & $50 \%$ & 3 & $20 \%$ & 7 & $30 \%$ \\
\hline No sabe no responde & 1 & $13 \%$ & 1 & $7 \%$ & 2 & $\%$ \\
\hline & 8 & $100 \%$ & 15 & $100 \%$ & 23 & $100 \%$ \\
\hline
\end{tabular}

En la tabla anterior se observa que el 30\% de las respuestas de los expertos consultados consideran que no ha habido ni avances ni retrocesos en la promoción de centros de investigación pendientes, en tanto que el 26\% respondió que había avances y retrocesos y un 22\% respondió que había retrocesos, el 27\% las respuestas de los expertos de la academia consideraron esta situación frente a un 13\% de las respuestas de los expertos del sector. Y el 13\% respondió que había avances, porcentaje que correspondió en su totalidad a las respuestas de los expertos de la academia. A continuación, se muestrab las acciones observadas en el 2019 por los expertos consultados en relación con la investigación en centros independientes de investigación: los expertos consideraron que, si bien algunas instituciones han promovido trabajos de investigación, estas no son un lineamiento ni una política organizacional, sino más una iniciativa de personas interesadas al interior de las organizaciones. 


\section{Escenario que enmarca la acción del sector cooperativo}

A continuación, se presenta el resultado de uno de los trabajos desarrollados en el grupo focal en que se le consultó a los expertos sobre la percepción que tenían sobre el cumplimiento de las variables estratégicas consideradas en Obregón-Puyana et al. (2014).

Tabla 12. Percepción cumplimiento de las variables estratégicas

\begin{tabular}{llll}
\hline & Variables estratégicas & SI & NO \\
\hline 1. & Incidencia en las políticas públicas & $17 \%$ & $83 \%$ \\
\hline 2. & Gestión gremial de la integración & $25 \%$ & $75 \%$ \\
\hline 3. & Identidad solidaria y de cooperación & $58 \%$ & $42 \%$ \\
\hline 4. & Vínculo asociativo & $42 \%$ & $58 \%$ \\
\hline 5. & Investigación sobre modelo cooperativo & $75 \%$ & $25 \%$ \\
\hline
\end{tabular}

De acuerdo con la tabla 12, las variables estratégicas 1, 2 y 4 no se están cumpliendo, si se considera que el cumplimiento de la variable queda expresado con la mayor percepción de los expertos. Del mismo modo, las variables que reflejan incumplimiento son la 3 y la 5, identidad solidaria y de cooperación e investigación sobre el modelo cooperativo. Vale la pena advertir que la percepción del grado de cumplimiento de la variable 3 es ligeramente inclinado al Sí, lo que no se puede establecer como concluyente, lo que sí se puede observar en la variable 5 donde la percepción de al menos $3 / 4$ partes de los expertos consultados considera que ha habido avances, por el mayor número de investigaciones, de publicaciones, investigadores y universidades interesadas en el tema, luego puede tener mayores posibilidades de cumplimiento, aunque en la actualidad no es procentaje que convenza al 100\% de los expertos. La siguiente tabla muestra el escenario en que se ubica el sector de acuerdo con la percepción de los expertos.

Tabla 13. Escenario encontrado de acuerdo con la percepción de los expertos

\begin{tabular}{lc}
\hline Variables estratégicas & Escenario \\
\hline Incidencia en las políticas públicas & 0 \\
\hline Gestión gremial de la integración & 0 \\
\hline Identidad solidaria y de cooperación & 1 \\
\hline Vínculo asociativo & 0 \\
\hline Investigación sobre modelo cooperativo & 1 \\
\hline
\end{tabular}




\section{Líneas de acción propuestas por los expertos para el logro de cada una de las variables estratégicas}

Frente a qué acciones se pueden desarrollar para el logro de las variables estratégicas planteadas, los expertos sugerían las siguientes:

\section{Incidencia en las políticas públicas}

- Todas las organizaciones públicas deben prepararse para la construcción de economías transformadoras que incluyan la economía social y solidaria.

- De la mano de la academia, el sector y sus organismos de integración deben desarrollar programas que consoliden bases de información confiable y el desarrollo de estudios de impacto para el desarrollo territorial, que acompañen procesos de evaluación de política pública.

- Promover, a través del vínculo con organismos internacionales, el conocimiento de cómo se resuelven los problemas en otros países lo que, probablemente, de luces de cómo intervenir los nuestros.

- Debe impulsarse la consolidación de un movimiento social y solidario para la construcción de política pública.

- Articular y consolidar las necesidades y proyecciones conjuntas del sector para defender en los espacios políticos.

- Promover la participación y la cultura política, pensamiento crítico social, solidaria y humanista en los asociados, para lograr mejorar la selección de quienes representan los intereses del sector en los organismos públicos de elección popular.

- Promover esquemas de supervisión en los que se otorgue mayor participación del sector sobre esta actividad. Es posible que, a mayor participación del sector, mayor responsabilidad y fortalecimiento tenga. Se puede pensar en procesos de supervisión auxiliar o autocontrol.

\section{Gestión gremial de la integración}

- Promover la integración de la economía informal y buscar su articulación con la economía social y solidaria, así como con otros sectores como las MiPymes y entre todos construir propuestas que busquen lograr mayor incidencia en las políticas públicas del orden nacional, regional y local.

- Lograr de la mano de la academia aplicar metodologías para el desarrollo de programas inclusivos. 
- Socialización constante de los avances de la economía social y solidaria, divulgación y utilización de los medios de comunicación.

- Promover alianzas para poner en marcha territorios solidarios.

- Promover un sistema de integración gremial incluyente. Reconocer la diversidad para una visión común de necesidades y soluciones. Impulsar diálogos responsables con los gremios de la economía social y solidaria.

\section{Identidad solidaria y de cooperación}

- Generar escenarios de formación y participación para organizaciones de base.

- Conocer, entender, comprender y estudiar el modelo solidario.

- Construir e implementar un modelo educativo solidario e innovador.

- Cuidar las prácticas y propuestas que sean pertinentes para la cultura e identidad solidaria.

- Fomento real de los principios y valores.

- Explicar, demostrar, la naturaleza no capitalista y solidaria del quehacer socio-empresarial.

- Fortalecer la identidad a través de los grupos de interés de las organizaciones solidarias.

\section{Vínculo asociativo}

- Utilizar los servicios del sector solidario.

- Crear redes permanentes de servicios solidarios entre organizaciones de la economía social y solidaria.

- $\quad$ Que el balance social sea usado como una herramienta de gestión en todas las organizaciones.

- Complementar la propuesta de balance social que hace la Superintendencia de la Economía Solidaria.

- Motivar y crear sentido de pertenencia.

\section{Investigación sobre el modelo cooperativo y solidario}

- A través de los centros y grupos de investigación, generar espacios de apropiación social de conocimiento.

- Realizar procesos de investigación aplicada articulada con varios actores. 
- Compartir metodologías y hallazgos de investigación para el desarrollo cooperativo y solidario.

- Las organizaciones de base deben reconocer la importancia de la investigación sobre procesos de desarrollo empresarial.

- Desarrollar material educativo en diferentes formatos que mejoren los procesos de gestión en las organizaciones.

- Promover estrategias, técnicas y metodologías para la creación y sostenibilidad del circuito económico solidario.

- Documentar las acciones que adelantan las organizaciones de la economía solidaria.

- Profundizar en investigaciones sobre balance social.

- Estudiar, crear y diseñar instrumentos e indicadores que permitan medir los beneficios de estar en una organización de la economía social y solidaria.

- Desarrollar investigaciones que contribuyan al fortalecimiento de diálogos, norte/sur y sur/sur.

- Vincular estudiantes de últimos semestres en prácticas solidarias.

- Impulsar proyectos conjunto entre universidades y grupos de investigación.

- Promover el desarrollo de trabajos de grado con investigaciones en temas de economía solidaria.

- Facilitar el estudio de los casos de éxito y fracaso.

- Consolidar un centro de estudio y pensamiento para el sector integrado.

- Identificar y desarrollar líneas de investigación en economía social y solidaria.

- Crear bancos de datos de Ecosol.

- Acercar el gremio a la academia.

\section{Discusión}

En esta sección se interpretan los datos en relación con la hipótesis planteada y el estado del conocimiento actual del tema estudiado. Por esta razón se considera que pese a que puede haber avances en la realización de acciones que propendan por la incidencia en las políticas públicas, por el fortalecimiento de la gestión gremial de la integración, la identidad solidaria y de cooperación, el vínculo asociativo y la investigación sobre el modelo cooperativo y solidario, aún faltan muchas acciones para lograr lo previsto en el estudio de Obregón-Puyana et al. (2014). También, se ratifica la validez de las variables estratégicas propuestas. A continuación, se presentan los elementos de discusión por cada una de estas variables estratégicas analizadas. 
Esta lectura debe hacerse teniendo presente y cruzando el diagnóstico realizado para cada variable en el documento publicado por el IEMP.

\section{Variable estratégica: Incidencia en las políticas públicas}

Si bien es cierto que se han producido algunos avances en cuanto a consensos regionales para formulación de política pública, como las ya mencionadas de Antioquia, Santander, Medellín, Cali y Bogotá, por ejemplo, la incidencia lograda no es significativa, ya que esta depende principalmente de la voluntad política de gobernantes locales y regionales ocasionales y no de manera permanente, ya que el desinterés del Estado y los gobiernos que se han instalado en estos años ha llevado a que no se actúe en consenso con el sector ni con la población para fijar la política pública. Los debates sobre los planes de desarrollo de los últimos gobiernos si bien plantean la participación de la población y sus representantes, sus opiniones y propuestas no son tenidas en cuenta. Sin embargo, la mayor preocupación es el retroceso que se está produciendo en relación con la negativa del actual gobierno para cumplir con el Acuerdo de Paz.

En lo que tiene que ver con la creación del Ministerio de Economía Social y Solidaria, no se ven avances de importancia, pues en el gobierno no se habla de esta propuesta y no hay ambiente en el Congreso ni consensos ni conocimiento en el sector sobre este tema. Pareciera haber más bien retrocesos significativos por parte del Estado. Ejemplo de ello es la oposición al Acuerdo de paz del actual gobierno y la pérdida de un departamento administrativo como Ministerio técnico como era Dansocial, reemplazado por una oficina en el Ministerio de Trabajo, la UAEOS, en el gobierno anterior.

Como se mencionó atrás, frente a las acciones que proponía el estudio realizado por el IEMP, se puede comentar que no se han tenido en cuenta, quedando pendiente su análisis y posible realización por parte del sector. La actividad más cercana fueron las realizadas para ofrecer un aporte al plan nacional de desarrollo (Confecoop, 2019), el avance en la consolidación de un grupo de congresistas (Confecoop, 2018; Asocooph, 2018) y las propuestas sobre el Plan de desarrollo y los esfuerzos para continuar con la idea de realizar mediciones del impacto que la economía solidaria y social tiene en el país. 


\section{Variable estratégica: Gestión gremial de la integración}

A pesar de que se han producido incipientes procesos de integración en el sector para tratar diferentes posiciones relacionadas con el Estado, en especial motivadas por el proceso relacionado con el Acuerdo de Paz, como han sido la Mesa nacional de ESS, las Mesas regionales de economía social y solidaria por la paz, los nodos de la Convergencia alternativa social y solidaria por la paz, los Encuentros nacionales de jóvenes y mujeres solidarios y las propuestas particulares impulsadas por Redess y apoyadas por algunas vertientes del sector, puede concluirse que estos esfuerzos han sido insuficientes, ya que se han dado de manera dispersa y centrados en algunas organizaciones solidarias de importancia, que refleja que no hay un verdadero interés y sentido de trabajar conjuntamente, demostrando una "debilidad de integración intergremial que refleja poco interés por adelantar acciones conjuntas en procura de propósitos más elevados y comprometidos para bien del sector" como se ha mencionado anteriormente.

En términos de actividades propias del sector se han dado algunos resultados a nivel regional mas no nacional y de nivel sectorial, como han sido el caso de las redes de servicios financieros Coopcentral - Visionamos, la Red servicios financieros Conecta, la red de intercooperación en Antioquia.

En el caso de la academia, se tiene una visión más optimista, ya que se han logrado la consolidación de las redes Unicossol y Ciriec-Colombia, realizando actividades de integración y vinculación de las diferentes vertientes del sector en las acciones que se desarrollan según sus planes anuales particulares, pero, paradójicamente, no se ha dado todavía un proceso de articulación entre las dos redes por causas inexplicables.

En relación con las acciones previstas por el estudio y como lo han mencionado los expertos consultados, se han producido intentos de promoción de ferias locales, regionales y nacionales con organizaciones solidarias, pero sin avances significativos en la idea de consolidar un sello de origen productivo solidario, que sigue siendo una interesante propuesta sin mayor desarrollo, contándose con un impulso que Agrosolidaria viene realizando en este sentido. El sello cooperativo existente desde hace más de una década se ha ido extinguiendo lánguidamente por el poco interés de las organizaciones y el gremio en fortalecerlo e impulsarlo. 


\section{Variable estratégica: Identidad solidaria y de cooperación}

Se han producido algunos avances en este sentido, tanto en acciones impulsadas por el Estado como por el sector y la academia, en especial, buscando promocionar servicios entre los asociados, destacándose principalmente en la actividad del ahorro y el crédito, aunque es preocupante el atraso tecnológico y la poca cultura digital desarrollada en el sector, ya que apenas se está "despertando" frente a la era digital que emerge.

A pesar de los esfuerzos por impulsar procesos educativos relacionados con la identidad y la particularidad del modelo que representa el sector, por parte del Estado, el sector y la academia, el avance entre las bases sociales es mínimo y predomina la visión de usuario y no de dueño, además de que hay una gran despreocupación por el proceso educativo, formador, capacitador e informativo que propone el principio solidario, lo que se traduce en bajos porcentajes de participación en los procesos electorales de las organizaciones y en la vida cotidiana de estas y en relacionamiento inter e intra bases sociales. Para las cooperativas, la captación de los recursos de educación por parte del Estado, crea un problema para continuar con los procesos educativos.

Se tiene una preocupación especial en cuanto al papel que juegan los delegados en cuanto ser transmisores de los sucesos que acaecen en las asambleas, pues no se convierten en retroalimentadores de estos hechos entre las bases sociales del modelo.

\section{Variable estratégica: Vínculo asociativo}

Como se mencionaba en la variable estratégica anterior, se puede considerar que el asociado sigue teniendo un vínculo muy débil con su organización y que actúa más como un usuario que como dueño, exigiendo servicios y beneficios, pero poco entendido en cuanto a sus deberes. En cuanto al balance social, se considera que hay un avance no solo por el hecho de que haya una propuesta que emerge desde la Superintendencia de la Economía solidaria, sino por los estudios que se viene realizando tanto por parte del Estado como por la academia, alrededor del tema de cómo medir el impacto que el sector genera en su base social y en la comunidad, a pesar de la deficiente estadística que existe para esta labor y la inexistencia de cuentas nacionales que reflejen el impacto del sector.

Se debe reconocer el esfuerzo que han hecho las organizaciones solidarias para tratar de desarrollar un balance social de carácter cualitativo y según los intereses de 
cada organización, aunque es preocupante la pérdida de importancia que tiene la consideración y concepción de la gestión social en muchas de las organizaciones solidarias, tanto por parte de directivos, dirigentes y gerentes como por parte de los asociados, que ha generado un desbalance en relación con la consideración del objetivo social y el objetivo económico, en detrimento de lo social.

\section{Variable estratégica: Investigación sobre modelo cooperativo}

Desde la perspectiva de los actores de la academia, hay una consideración favorable en cuanto a considerar los avances logrados que se manifiestan tanto en el número creciente de profesores dedicados al tema, grupos de investigación conformados, líneas de investigación planteadas, proyectos de investigación realizados y la producción generada, como lo evidencian los estudios hechos (Cueto Fuentes, Arboleda Álvarez y Zabala Salazar, 2018; Davila, 2018), a pesar del ambiente negativo y reticente que tiene la academia tradicional en relación con el tema.

I gualmente, la presencia de Doctores titulados y doctorandos alrededor del tema de la economía social y solidaria, así como la consolidación de las redes Unicossol y Ciriec-Colombia y el desarrollo de sus planes de acción en cuanto a proyectos realizados y actividades locales, regionales, nacionales e internacionales de difusión de resultados de investigación y de interacción con pares internacionales y nacionales, fundamenta la visión positiva que se tiene.

Sin embargo, es preocupante el desconocimiento y lejanía que tiene el gremio y muchas de las organizaciones solidarias alrededor estos avances, como se pudo observar en algunas de las respuestas dadas por los actores del sector y las organizaciones al responder al instrumento aplicado y al ejercicio del taller desarrollado. Preocupa, igualmente, el papel del Estado en dotar al sector de una estadística confiable y profunda, así como la inexistencia de un censo del sector que dé cuenta creíble de su realidad, más allá de las cifras contables y financieras de las cuales se dispone.

\section{Conclusiones}

A manera de conclusión se observa la validez de las variables estratégicas identificadas por Obregón-Puyana et al. (2014), el sector cooperativo y solidario se ubica en el escenario denominado en ese trabajo "Situación de confort hacia la desaparición", en el que, a pesar de la existencia de avances, no son suficientes para que la evolución de las variables estratégicas satisfaga las hipótesis planteadas. Pareciera que 
los actores se encontraran en una zona de confort sin que se quisiera salir de allí, ello podría conducir, en el peor de los casos, a la desaparición del sistema de Economía Solidaria. La situación del escenario es que el sector cooperativo y solidario está condicionado por las crisis cíclicas de la economía capitalista, que tiene implicaciones de distinto orden para el ser humano y la sociedad, sin que asuma las riendas de su propio destino.

De todos modos, producto de las crisis constantes a nivel nacional e internacional, la sociedad civil organizada a escala mundial y local obligará la emergencia de un sector capaz de contraponerse a las decisiones del Estado y las externalidades negativas del mercado creado a partir del ordenamiento de la economía capitalista, donde sería impredecible el futuro de los actores actuales del sector cooperativo y solidario, por lo que es de suma importancia asumir las riendas de su destino y hacerse participe en la forma como se va desarrollando el mundo.

Frente a las acciones a seguir, se sugiere tener en cuenta las planteadas en el documento de Obregón-Puyana et al. (2014), orientadas a unos objetivos estratégicos allí planteados. Del mismo modo las acciones propuestas por los expertos y presentadas en este artículo, son importantes de considerar en cualquier actividad que se proponga desarrollar en beneficio de quienes hacen parte de la economía social y solidaria. De todas estas acciones, a continuación, se resaltan algunas que son especiales para el logro de las variables estratégicas planteadas:

\section{Incidencia en las políticas públicas}

- Preparación, integración, educación para el desarrollo de planes y programas en que se articule la economía social y solidaria, el sector público y la academia.

- Consolidar bases de información confiables que se articulen a nivel nacional, regional y local.

\section{Gestión gremial de la integración}

- Promover la integración de la economía informal y buscar su articulación con la economía social y solidaria, así como con otros sectores como las MiPymes y entre todos construir propuestas que logren mayor incidencia en las políticas públicas del orden nacional, regional y local. 


\section{Identidad solidaria y de cooperación}

- Explicar, demostrar y poner en práctica la naturaleza no capitalista, pero sí solidaria del quehacer socio-empresarial de la economía social y solidaria.

\section{Vínculo asociativo}

- Crear redes permanentes de servicios solidarios entre organizaciones de la economía social y solidaria.

- $\quad$ Que el balance social sea usado como una herramienta de gestión en todas las organizaciones.

\section{Investigación sobre el modelo cooperativo y solidario}

- Promover estrategias, técnicas y metodologías para la creación y sostenibilidad del circuito económico solidario.

\section{Referencias}

Álvarez Arévalo, J. y Benavides Carrascal, E. (2018). Análisis estratégico y prospectivo del sector solidario del municipio de Ocaña, hacia el año 2034. Universidad Nacional Abierta y a Distancia (UNAD).

Bitar, S. (2015). América latina debe pensar en el futuro. En G. Baena Paz, Planeación prospectiva estratégica teorías, metodologías y buenas prácticas en América Latina (pp. 53-68). Universidad Nacional Autónoma de México.

Confecoop (2019). Propuesta para el plan nacional de desarrollo pacto por la economía solidaria y cooperativa. https://confecoop.coop/actualidad/actualidad-2019/propuesta-para-el-plan-nacional-de-desarrollo-pacto-por-la-economia-solidaria-y-cooperativa

Confecop (2018). Comunicoop 2262. Bancada cooperativista y Junta Directiva de Confecoop analizan efectos de ley de financiamiento para las cooperativas. https://confecoop.coop/ comunicoop/2018/comunicoop-2262-bancada-cooperativista-y-junta-directiva-de-confecoop-analizan-efectos-de-ley-de-financiamiento-para-las-cooperativas/ 
Cueto Fuentes, E., Arboleda Álvarez, O. y Zabala Salazar, H. (2018). Una década de economía social y solidaria en Colombia: análisis de la producción investigativa y académica 2005-2015. Corporación Universitaria Minuto de Dios y Universidad Católica Luis Amigó.

Dávila, R., Vargas, A., Blanco, L., Roa, E., Cáceres, L. y Vargas, L. (2018). Características de la economía solidaria colombiana. Aproximaciones a las corrientes influyentes en Colombia. CIRIEC España, Revista de Economía Pública, Social y Cooperativa, 93, 85-113, https://doi. org/10.7203/CIRIEC-E.93.10327

Godet, M. (1999). De la anticipación a la acción. Manual de prospectiva y estrategia.: Alfomega.

Henao V., L. M, V. (2015). Prólogo. En G. Baena Paz, Planeación prospectiva estratégica teorías, metodologías y buenas prácticas en América Latina (pp. 23-28). Universidad Nacional Autónoma de México.

Mera Rodríguez, C. (2014). Pensamiento prospectivo. Visión sistémica de la construcción del futuro. Análisis: revista colombiana de humanidades, 84, 89-104.

Mojica, F. (2005). La construcción del futuro. Concepto y modelo de prospectiva estratégica, territorial y tecnológica. Convenio Andrés Bello-Universidad Externado de Colombia.

Obregón-Puyana, J., Castillo, D., Rodríguez, N., Sánchez, C. y Mora, C. (2014). Aplicación del modelo prospectivo estratégico al sector cooperativo colombiano; horizonte 2031. Instituto de Estudios del Ministerio.

Peralta Quevedo, S. y Herrera Niño, L. (2012). Estado del arte. Estudio prospectivo estratégico para el sector solidario en el departamento del Meta hacia el año 2022. Universidad Nacional Abierta y a Distancia (UNAD).

Quiroga, M. (2018). Asocooph. Creada en el Congreso la Comisión Accidental de Cooperativismo y Economía Solidaria. https://repository.ucc.edu.co/bitstream/20.500.12494/7067/4/2018_ asociacion_cooperativas_empresas.pdf

Rodero, T. (2015). Perspectivas de la prospectiva. Un diálogo de saberes sobre el porvenir. En G. Baena Paz, Planeación prospectiva estratégica teorías, metodologías y buenas prácticas en América Latina (pp. 217-250). Universidad Nacional Autónoma de México.

Rosa, P. C. (2016). Los caminos de la utopía: enfoques y perspectivas del campo de la Economía Social. Cooperativismo \& Desarrollo, 24(109). https://doi.org/10.16925/co.v24i109.1512 
32 Buscando los caminos del sector cooperativo y solidario

Serna Gómez, H. H. y Rodríguez Barrero, M. S. (2016). El sector solidario como alternativa para el desarrollo social e inclusivo en el postconflicto colombiano. Cooperativismo \& Desarrollo, 23(107). https://doi.org/10.16925/co.v23i107.1250 